CERN-TH.6834/93

LPTENS $93 / 7$

\title{
Rational Theories of 2d Gravity from the Two-Matrix Model
}

\author{
J.-M. Daul \\ Laboratoire de Physique Théorique \\ Département de Physique de l'Ecole Normale Supérieure \\ 24 rue Lhomond, 75231 Paris Cedex 05, France, \\ V.A. Kazakov \# and I. K. Kostov * \\ Theoretical Physics Division, CERN \\ CH-1211 Geneva 23, Switzerland
}

The correspondence claimed by M. Douglas between the multicritical regimes of the two-matrix model and $2 \mathrm{~d}$ gravity coupled with $(p, q)$ rational matter field, is worked out explicitly. We found the minimal $(p, q)$ multicritical potentials $U(X)$ and $V(Y)$, which are polynomials of degree $p$ and $q$, correspondingly. The loop averages $W(X)$ and $\tilde{W}(Y)$ are shown to satisfy the Heisenberg relations $\{W, X\}=1$ and $\{\tilde{W}, Y\}=1$ and essentially coincide with the canonical momenta $P$ and $Q$. The operators $X$ and $Y$ create the two kinds of boundaries in the $(p, q)$ model related by the duality $(p, q) \leftrightarrow(q, p)$. Finally, we present a closed expression for the two two-loop correlators, and interpret its scaling limit.

Submitted for publication in: Nuclear Physics B

CERN-TH.6834/93

March 1993

\# Permanent address: Laboratoire de Physique Théorique, Département de Physique de l'Ecole Normale Supérieure, 24 rue Lhomond, 75231 Paris Cedex 05, France

* Permanent address: Service de Physique Théorique de Saclay, CE-Saclay, F-91191 Gif-surYvette Cedex, France 


\section{Introduction}

The theory of non-critical strings, or $2 \mathrm{~d}$ gravity with central charge of the matter $c \leq 1$, has made great steps forward in the last years. This theory allows two very different formalisms: the Liouville theory [1] and the two dimensional Regge calculus based on matrix models [2], [3], [4], which are in a sense complementary to each other. Spectacular results in calculations of critical exponents [5], [6], [7], [8], [9], [10], macroscopic observables [11], [12], [13] and correlation functions [14], [15], [16] have been accomplished. One of the important achievements was a non-perturbative analysis of $2 \mathrm{~d}$ gravity in the framework of the double scaling limit [17], which found its most elegant realization in the KdV hierarchy of string equations [18].

The generic rational string theory can be constructed as a chain of coupled random matrices [18], [19]. However, one can look for a more economic way to do it. In this paper we address the problem of finding the simplest model of (discretized) 2d gravity with matter implementing the whole physics of non-critical strings; or, in other words, finding the simplest matrix model containing all known critical regimes corresponding to $(p, q)$ conformal matter.

The first attempt to build such a minimal description was made in ref. [20], where various matters coupled with gravity were generated as multicritical regimes of the onematrix model. Since the random surface representation (Feynman graphs) of this model does not contain any explicit introduction of matter (like spins at the sites of the random lattice) this latter appears as the multicritical excitations of "pure gravity" modes. It soon became clear [21], [22] that the multicritical regimes in the 1-matrix model correspond only to the special case $(q=2, p=2 n-1)$.

The next simplest possibility is the two-matrix model first introduced in [23] and solved in the large $N$ limit in [24]. As was pointed out by M.Douglas [25], one can get, by appropriately adjusting the potentials of both matrices, the critical regimes corresponding 
to various $(p, q)$-rational string theories, which in turn correspond to $2 \mathrm{~d}$ gravity interacting with matter fields having central charge

$$
c=1-6 \frac{(p-q)^{2}}{p q} .
$$

Some examples of this conjecture for particular values of $p$ and $q$ were considered in [26].

Technically, this means that in the realization of the Heisenberg algebra $[P, X]=1 / N$ in the basis of orthogonal polynomials of the two-matrix model [24], [27], the operators $P, X$ are the infinite matrices containing enough diagonals (unlike those in the one-matrix model) to describe the differential operators of a generalized KdV hierarchy of an arbitrary order. The appearance of generalized KdV flows in the two-matrix model was studied in ref. [28].

In this paper, we present the explicit construction of the $(p, q)$ critical points of the twomatrix model. The deviation from the critical point is due to the shift of the "cosmological constant" from its critical value $\mathrm{Q}$, while the matter remains critical. Consider for example the point $(3,4)$ describing the Ising model on a random graph [5]. The critical potential means that we are exactly at the spin ordering phase transition point, and by shifting the cosmological constant (the overall coupling in the matrix model) from its critical value we make the volume of the system big but finite. The existence of an explicit and relatively simple formula for the $(p, q)$ multicritical potentials explains why one was able to find explicitly the critical "temperatures" in many particular cases, such as Ising models on various types of random graphs.

We start from the equations of motion, relating the canonical momenta $P, Q$ to the coordinates $X, Y$, as they were derived (but not extensively used) in 25]. We wrote them

1 Strictly speaking, in the case of a non-unitary critical point $p>q+1$ the coupling of the matrix model is not the cosmological constant. The latter is coupled with the identity operator, which in this case is not the operator of minimal dimension. We will discuss this point later. 
in an operator form, valid for any $N$ but adjusted to the double scaling limit. The method looks quite promising for more complicated matrix models (in [29] it was applied to the so-called induced gauge theory).

We extract the critical potentials for the $(p, q)$ critical regime from the algebraic form of the equations of motion. In order to achieve the $(p, q)$ critical point it is sufficient to take as the potentials $U(X)$ and $V(Y)$ two polynomials of degree $p$ and $q$, respectively: this allows for easier, more explicit and more general formulae than in ref.[30]. For investigating the one-loop averages, we exploit the Heisenberg algebra for $[P, X]=1 / N$, which appears in the formalism of orthogonal polynomials. In the large $N$ (classical, planar) limit, the commutator reduces to the Poisson bracket $\{P, X\}$ of the corresponding "classical" functions. The most important observation here, from our point of view, is that the "KdV-momentum" $P$ is equal to the 1-loop correlator $W(x)=\left\langle\frac{\operatorname{Tr}}{N} \frac{1}{x-\hat{X}}\right\rangle$ (up to some part, independent from the cosmological constant, giving no contribution to the Poisson brackets). This is true even before the continuous limit, and it gives a direct physical meaning to the Heisenberg relation: $\{W, X\}=1$, allowing the direct calculation of the macroscopic observable $W(x)$.

Using all this we were able to rederive in a simple way the results of [11], [12] for $W(x)$ in the general $(p, q)$ case. The unitary $(m+1, m)$ series can be realized in two different ways. The first is just the general $(p, q)$ solution with $p=m+1, q=m$. The second way is to look for criticality under the condition that the two potentials $U$ and $V$ are equal. These two realizations turn to be physically very different.

In the non-symmetric realization the operators $X$ and $Y$ have different scaling dimensions and create two different kinds of boundary. Our conjecture is that they correspond to the Dirichlet and Neumann boundary conditions in the statistical realization of the $(m+1, m)$ model. The symmetric realization can be interpreted as a $m$ multicritical regime of the Ising model on a random graph. Then the operators $X$ and $Y$ are related by the $\mathbb{Z}_{2}$ symmetry in a very simple way. 
The situation is more complicated for the non-unitary cases; what we called, in the unitary case, the "cosmological constant" $\lambda$ (the overall constant in front of the matrix potential) couples there with an operator of lowest negative scaling dimension $\Delta_{-}=$ $\frac{1-|p-q|}{p+q-1}$ 22]. The genuine cosmological constant $\Lambda$ is coupled to the unit operator and measures the total volume of the random graph. These two constants are related by

$$
\lambda=\Lambda^{\frac{p+q-1}{2 q}} .
$$

In a recent paper [31] the general solution of dispersionless Heisenberg equations is given in terms of the $\tau$-function of the Whitham hierarchy. This "solution" is not very explicit, but it provides a general description of the vicinity of the critical point. Therefore we give a presentation of our results from the point of view of ref. 31].

Finally, we found the general expression for all two-loop correlators and investigated their scaling limit, which coincided with the known ones [11], [12]. In the scaling limit we observed a remarkable factorization property, inspiring a topological field theory interpretation. It generalizes the formula obtained in [32] in the case of the one-matrix model.

This paper is organized as follows:

In section 2 we formulate the model, and using the orthogonal polynomials formalism, derive the general equations of motion and their planar limit.

In section 3 we introduce the Heisenberg algebra for the matrix eigenvalue operator and its momentum, obtain their planar limit in terms of the Poisson brackets and establish the equality between the one-loop average and the Heisenberg momentum.

In section 4 an explicit formula for the $(p, q)$ multicritical potentials in the two-matrix model will be derived.

In section 5 we demonstrate how the equations of motion work on some examples of unitary as well as non-unitary theories.

In section 6, using the scaling limit of the classical Heisenberg relation (dispersionless $\mathrm{KdV}$ ) we reproduce the known results for the different loop correlators. 
In section 7 we discuss the vicinity of the critical point, in view of our interpretation of the loop average as the classical canonical momentum. We review the operator content of the theory.

In section 8 we interpret the two kinds of boundaries in the model as the Dirichlet boundaries in the two alternative standard realizations of the $(p, q)$ critical point with $q-1$ and $p-1$ spins.

In section 9 the two-loop correlator is calculated explicitly in the two-matrix model, and its scaling limit is found to be consistent with the known results.

In section 10 we summarize the results for the $\mathbb{Z}_{2}$-symmetric two-matrix model describing the unitary series $(m+1, m)$.

\section{Orthogonal polynomials in the two-matrix model with two arbitrary po- tentials}

The partition function for the two-matrix model is defined as an integral over two $N \times N$ hermitean matrices $X$ and $Y$

$$
Z=\int d^{N^{2}} X d^{N^{2}} Y e^{\beta \operatorname{Tr}(-U(X)-V(Y)+X Y)}
$$

where $U$ and $V$ are arbitrary polynomial (or even non-polynomial) potentials:

$$
U(X)=\sum_{n=1}^{p} \frac{g_{n}}{n} X^{n}, V(Y)=\sum_{n=1}^{q} \frac{h_{n}}{n} Y^{n} .
$$

The linear change of variables $X \rightarrow A_{1} X+A_{2}, Y \rightarrow A_{3} Y+A_{4}$ has a trivial Jacobian and preserves the form of the potential. Therefore the problem depends on $p+q-3$ relevant directions in the space of parameters $h_{1}, \ldots, h_{q}, g_{1}, \ldots, g_{p}, \beta$ in (2.2).

As was stated in [5], the Feynman graph expansion of this model, with respect to the non-quadratic terms in the potentials of (2.1), can be interpreted as the sum over graphs of partition functions of Ising spins placed in the vertices of these graphs. Since the Ising model in two dimensions describes fermions, and the sum over Feynman graphs 
can be interpreted as the functional integral over the two dimensional metric, it can be concluded that we deal with fermions interacting with $2 \mathrm{~d}$ gravity. As is well known, the $1 / N$ expansion allows the classification of these partition functions according to genus, so that the total partition function can be viewed as the functional integral of the fermionic string field theory with $1 / N$ as a string coupling. So in the limit $N \rightarrow \infty$ we are left with only planar $2 \mathrm{~d}$ manifolds.

On the other hand, the representation of $2 \mathrm{~d}$ manifolds in terms of planar Feynman graphs depends on the couplings in the potentials (2.2). One can perform the discretization by choosing either $\phi^{3}$ - or any $\phi^{n}$-potential, or even any mixture of vertices of different orders. As in the one-matrix model [20], one can adjust the corresponding couplings so as to get different multicritical behaviours, corresponding, in the two-matrix case, to all possible $(p, q)$ minimal models coupled with $2 \mathrm{~d}$ gravity. One can say that (unlike the case of SOS matter [19]) the $(p, q)$ matter is produced, in the two-matrix model, as the collective excitations of the $Z_{2}$ (Ising) degrees of freedom and those of the discretized metric of the world-sheet.

Let us start with briefly reviewing the method of diagonalization due to orthogonal polynomials. If we integrate with respect to the angular degrees of freedom, (2.1) becomes an integral over the $2 N$ eigenvalues $x_{1}, \ldots, x_{N}, y_{1}, \ldots, y_{N}$ :

$$
Z=\int \prod_{i=1}^{N} d x_{i} d y_{i} \Delta(x) \Delta(y) e^{\beta \sum_{i=1}^{N}\left(-U\left(x_{i}\right)-V\left(y_{i}\right)+x_{i} y_{i}\right)}
$$

We introduce the orthogonal polynomials $|n\rangle=\zeta_{n}(x)$ and $\langle n|=\eta_{n}(y)$ by the orthonormality relation

$$
\langle m \mid n\rangle=\int d x d y e^{\beta(-U(x)-V(y)+x y)} \zeta_{m}(x) \eta_{n}(y)=\delta_{n, m}
$$

We denote the matrix elements:

$$
X_{m n}=\langle m|x| n\rangle, \quad Y_{m n}=\langle m|y| n\rangle, P_{m n}=\left\langle m\left|\frac{\partial}{\partial x}\right| n\right\rangle, \quad Q_{m n}=\left\langle m\left|\frac{\partial}{\partial y}\right| n\right\rangle
$$


The following "equations of motion" are easily obtained by doing an integration by parts:

$$
\frac{1}{\beta} P_{m n}=\left\langle m\left|U^{\prime}(x)\right| n\right\rangle-Y_{m n}, \quad \frac{1}{\beta} Q_{m n}=\left\langle m\left|V^{\prime}(y)\right| n\right\rangle-X_{m n}
$$

At this point it is useful, with a view to the later introduction of differential operators in the double scaling limit, to change the notations for the indices of the matrix elements:

$$
X_{k}(n)=X_{n-k, n}, \quad Y_{k}(n)=Y_{n, n-k}
$$

Now $n$ marks the position of the matrix element on the diagonal, and $k$ is its deviation from it. Then the action of the operators $X, Y$ on the orthogonal polynomial basis is described by the formulae

$$
X|n\rangle=\sum_{k=-1}^{q-1} X_{k}(n)|n-k\rangle ; \quad\langle n| Y=\sum_{k=-1}^{p-1} Y_{k}(n)\langle n-k| .
$$

To prepare for both the continuum and planar limit, introduce the continuous variables

$$
t=n / \beta, \quad \lambda=N / \beta,
$$

the operator $\hat{n}$ whose eigenvalues are $n$ (i.e. $\hat{n}|n\rangle=t|n\rangle, t=n / \beta$ ), and the conjugate coordinate $\omega$, as it was prescribed in ref. [33]. Then $\hat{n}$ is represented by the differential operator $\hat{n}=-(1 / \beta) d / d \omega$, where $\beta=\frac{N}{\lambda}$. The constant $1 / \beta$ plays the role of the Planck constant in this formalism. The operators $X$ and $P$ are represented by the functions

$$
X(\omega, t)=\sum_{k=-1}^{q-1} e^{k \omega} X_{k}(n), P(\omega, t)=\sum_{k=1}^{(q-1)(p-1)} e^{k \omega} P_{k}(n) ; \quad t=n / \beta
$$

Similarly one defines the functions $Y(\omega, t), Q(\omega, t)$ representing the operators $Y$ and $Q$

$$
Y(\omega, t)=\sum_{k=-1}^{p-1} e^{k \omega} Y_{k}(n), Q(\omega, t)=\sum_{k=1}^{(p-1)(q-1)} e^{k \omega} Q_{k}(n) ; \quad t=n / \beta
$$

It is always possible to normalize the polynomials (2.4) so that

$$
Y_{-1}=1, Q_{1}=t
$$


Then the coefficient $X_{-1}$ is determined as a function of $t=\lambda$ :

$$
X_{-1}(n)=R(t), \quad P_{1}(n)=\frac{t}{R(t)}
$$

where $R(t)$ is related to the susceptibility

$$
R(\lambda) \sim \frac{\partial^{2}}{\partial \lambda^{2}} \log Z
$$

Note that for any fixed highest power of the polynomial potentials (2.2), we have fixed highest powers with respect to $z=e^{\omega}$ in (2.10) and (2.11).

Now eqs. (2.6) read

$$
\begin{aligned}
& P(\omega, t)=U^{\prime}\left(X\left(\omega, t-\frac{1}{\beta} \frac{\partial}{\partial \omega}\right)\right) \cdot 1-Y\left(-\omega+\frac{1}{\beta} \frac{\partial}{\partial t}, t\right) \cdot 1 \\
& Q(\omega, t)=V^{\prime}\left(Y\left(\omega, t-\frac{1}{\beta} \frac{\partial}{\partial \omega}\right)\right) \cdot 1-X\left(-\omega+\frac{1}{\beta} \frac{\partial}{\partial t}, t\right) \cdot 1
\end{aligned}
$$

The compatibility of eqs. (2.15) with the condition that $P(\omega, t)$ contains only positive powers of $e^{\omega}$ lead, in the limit $N \rightarrow \infty$, to a triangular system of algebraic equations for the coefficients $X_{k}$ and $Y_{k}$ in the expansions (2.10) and (2.11).

The operator equations (2.15) are exact and valid for any $N$. They are very useful to go to the planar limit or to the double scaling limit. In the planar limit, we have to drop all the derivatives in the arguments of the functions $X, Y$ in $(2.15)$, and we arrive to the following system of (algebraic) equations:

$$
\begin{aligned}
& P(\omega, t)=U^{\prime}(X(\omega, t))-Y(-\omega, t) \\
& Q(\omega, t)=V^{\prime}(Y(\omega, t))-X(-\omega, t) .
\end{aligned}
$$

Again, the condition that $P, Q$ have only positive powers of $e^{\omega}$ is sufficient to find all the functions $P, Q, X, Y$.

In the symmetric case $U=V$ the two equations (2.16) reduce to a single equation for $P(\omega, t)=Q(\omega, t)$ and $X(\omega, t)=Y(\omega, t)$

$$
P(\omega, t)=U^{\prime}(X(\omega, t))-X(-\omega, t)
$$


where, choosing the symmetric normalization of the polynomials,

$$
P=\frac{t}{\sqrt{R}} e^{-\omega}+\ldots, X=\sqrt{R} e^{\omega}+\ldots
$$

This equation allows the coefficients $X_{k}$ and $P_{k}$ to be expressed in terms of the potential $U$. Once the potential is fixed, the first coefficient $R$ is found as a function of $t=\lambda$. For the $(p, q)$-critical potential it behaves as $R \sim \lambda^{2 / p+q-1}+$ const.

The explicit expression of $X$ and $P$ can be found more easily by means of the canonical commutation relations, to be studied in the next section.

\section{Heisenberg algebra, its classical limit and loop correlators in the planar limit}

It is obvious from the definitions (2.5) of the operators $X, Y, P, Q$ that they obey the Heisenberg commutation relations:

$$
[P, X]=\frac{1}{N},[Q, Y]=\frac{1}{N}
$$

In the classical limit $N \rightarrow \infty$ the commutator in eq. (3.1) is replaced by the Poisson bracket

$$
\{P, X\}=\frac{\partial P}{\partial t} \frac{\partial X}{\partial \omega}-\frac{\partial P}{\partial \omega} \frac{\partial X}{\partial t}=1
$$

Let us now consider the loop average

$$
W(x, \lambda)=\left\langle\frac{\operatorname{Tr}}{N} \frac{1}{x-\hat{X}}\right\rangle
$$

as a function of $\omega$ and $\lambda$ through $x=X(\omega, \lambda): W=W(X(\omega, \lambda), \lambda)$. We will show that this function has the same Poisson bracket with the coordinate $X(\omega, \lambda)$ as the momentum $P(\omega, \lambda)$. By definition of the average in the basis of orthogonal polynomials:

$$
\frac{\partial}{\partial \lambda} W_{1}(x, \lambda)=\frac{1}{2 \pi i} \oint d \omega \frac{1}{x-X(\omega, \lambda)}=\left(\frac{1}{\left.\partial \omega X\right|_{\lambda}}\right)_{X(\omega, \lambda)=x}=\left.\frac{\partial \omega}{\partial X}\right|_{\lambda} .
$$


On the other hand,

$$
\left.\frac{\partial W}{\partial \lambda}\right|_{X}=\left.\frac{\partial W}{\partial \lambda}\right|_{\omega}-\left.\frac{\partial W}{\partial \omega}\right|_{\lambda} \frac{\left.\partial_{\lambda} X\right|_{\omega}}{\left.\partial_{\omega} X\right|_{\lambda}}=\left.\frac{\partial \omega}{\partial X}\right|_{\lambda}\{W, X\},
$$

which implies

$$
\{W(\omega, \lambda), X(\omega, \lambda)\}=1
$$

Similarly, for

$$
\tilde{W}(y, \lambda)=\left\langle\frac{\operatorname{Tr}}{N} \frac{1}{y-\hat{Y}}\right\rangle
$$

we obtain

$$
\{\tilde{W}(\omega, \lambda), Y(\omega, \lambda)\}=1
$$

Equations (3.6) and (3.8) mean that

$$
\begin{aligned}
& W(\omega, \lambda)=P(\omega, \lambda)+f(x) \\
& \tilde{W}(\omega, \lambda)=Q(\omega, \lambda)+g(x),
\end{aligned}
$$

where the functions $f(x)$ and $g(x)$ do not depend on $\lambda$. Indeed, the explicit expression for $P$ as a Chebyshev polynomial [14], [34] coincides, as a function of $X$, with the expressions of the Wilson loop found in [1], [35].

Thus the classical Heisenberg algebra (3.6) and (3.8) is now formulated in terms of the basic macroscopic observables, the loop averages.

Equation (3.4), which can be written as

$$
\left.\frac{\partial P}{\partial \lambda}\right|_{X}=\left.\frac{\partial \omega}{\partial X}\right|_{\lambda}
$$

implies that there exists a "potential" $S$ such that

$$
P=\frac{\partial S}{\partial X}, \omega=\frac{\partial S}{\partial \lambda}
$$

The differential of this function is

$$
d S=P d X-\omega d \lambda
$$


The function $S(X, t)$ makes sense of the action of the Hamiltonian system, as a function of the coordinate $X$ and the time $t=\lambda$ along the classical trajectories.

It follows from (3.10), (3.3) and (3.12) that the function $S$ may be obtained as the average

$$
S(x, \lambda)=\left\langle\frac{\operatorname{Tr}}{N} \log (x-\hat{X})\right\rangle .
$$

Similarly, we could start with the $y$ variable and the action

$$
\tilde{S}(y, \lambda)=\left\langle\frac{\operatorname{Tr}}{N} \log (y-\hat{Y})\right\rangle,
$$

which is, in a certain sense, dual to (3.14). Indeed, by the equations of motion (2.16) the canonical pair of variables $(Q, Y)$ is obtained from $(X, P)$ by the involution $\omega \rightarrow-\omega$.

The relations of the type (3.6) found in this section are, of course, valid far beyond the two-matrix model, since the proof (3.4) and (3.5) is general enough. It is certainly true for the one-matrix model, for the multi-matrix chain and for any matrix model where the orthogonal polynomial formalism is available. One can even contemplate that these formulae are not specific to the orthogonal polynomial representation, and may be, some general approach to the string theories could be based on it.

\section{Explicit $(p, q)$-multicritical potentials of the two-matrix model}

The first step when investigating the $(p, q)$ critical behaviour of the two-matrix model is to find a set of critical couplings $g_{k}, h_{k}$. Near such a point in the space of couplings the size of the planar graphs tends to infinity and the corresponding $(p, q)$ "matter" exhibits long-range fluctuations. There are infinitely many ways to achieve the $(p, q)$ critical regime by varying the coupling constants; we choose the most economic option, which corresponds to potentials $U$ and $V$ of minimal power $p$ and $q$. Even this restriction does not fix the multicritical potential uniquely. We take advantage of the residual freedom to fix potentials with all coefficients rational. 
At the critical point $\lambda=\lambda^{*}$ one expects the following singular behaviour of $P$ and $X$

$$
X-X^{*} \sim \omega^{q}, \quad P-P^{*} \sim\left(X-X^{*}\right)^{p / q}=\omega^{p} \quad\left(e^{\omega}=z\right)
$$

Inserting this in (2.16), we see that the singular behaviour of $Q$ and $Y$ can only be

$$
Y-Y^{*} \sim \omega^{p}, \quad Q-Q^{*} \sim \omega^{q}=\left(Y-Y^{*}\right)^{q / p}
$$

The singular behaviour (4.1) and (4.2) is sufficient to fix the form of the functions (2.10) and (2.11) (we normalize $X$ so that $R=1$ )

$$
X_{*}(z)=\frac{(1-z)^{q}}{z}, \quad Y_{*}(z)=\frac{(1-z)^{p}}{z}
$$

The formal solution of (2.16) in terms of contour integration around $z=0$ gives the $(p, q)$ multicritical potentials

$$
\begin{aligned}
U_{p, q}^{\prime}(\xi) & =-\frac{1}{2 \pi i} \oint \frac{Y_{*}(1 / z)}{\xi-X_{*}(z)} d X_{*}(z) \\
V_{p, q}^{\prime}(\eta) & =-\frac{1}{2 \pi i} \oint \frac{X_{*}(1 / z)}{\eta-Y_{*}(z)} d Y_{*}(z)
\end{aligned}
$$

Here the contour of integration excludes all the poles except that at $z=0$.

Note that only the negative powers of $z$ are relevant for the definition of the potential from the given $X_{*}(z)$ and $Y_{*}(z)$, therefore $P(z)$ and $Q(z)$ do not enter the final expressions (4.4) . It is easy to see that the formulae (4.4)indeed give the polynomials of the p-th and q-th degree, respectively.

The unitary series of multicritical points $p=m+1, q=m$ can be achieved also for equal potentials $U=V$. If we take $X_{*}(z)=Y_{*}(z)=(1-z)^{m} / z$, then the $m$-critical potential given by (4.4) will describe the point $(m, m+1)$ for $m=3,4, \ldots$. For example, the case $m=3$ will give the Ising model on a 3-coordinate random lattice (with tadpole and self-energy subgraphs included).

The multicritical potentials for a few lowest $p$ and $q$, as well as for the symmetric case for a few lowest $m$, are presented in Appendix A. The same calculation can be carried out 
in the frequently used case of the potentials containing only even powers of matrices $X$ and $Y$. The details are presented in section 10. In Appendix A the results for the few first $m$ 's in the $Z_{2}$-symmetric case $U=V$ are presented.

A remarkable feature of this symmetric realization is that for every even $m$ the corresponding multicritical potential is stable (the coefficient in front of the highest power is positive), which provides a proper and quite economical way to non-perturbatively define the double scaling limit of the model.

\section{Some examples}

Should we try to find the classical functions $X(\omega, \lambda), Y, P, Q$ when given the potentials, we would have to solve a triangular system of algebraic equations, with degrees as high as $p$. On the other hand, expressing the potentials in terms of the coefficients of $X$ is fairly easy, as it involves only linear equations. Having recognized the critical form of $X, Y, \ldots$ (which are polynomials, up to some $1 / z$ term), we find critical potentials, and we can then try to move away from the critical point in the coupling constants space and see how $X, Y, \ldots$ have to be shifted : this can be done perturbatively, for any degree. In this way, we may access the scaling behaviour of our macroscopic observables, and check whether it satisfies the Heisenberg relation.

We shall first consider the symmetric case : the two coordinates are equal, and the one-loop correlator is given by the classical momentum (up to some function of $X$ ). Here, we reproduce the $(m, m+1)$ unitary case, with :

$$
\left\{\begin{array}{l}
X_{*}(z) \sim(1-z)^{m} \\
W_{*}(z) \sim(1-z)^{m+1}
\end{array}\right.
$$

The corresponding critical behaviour appears when we shift $\lambda$ away from its critical value: this is a particular move in the space of parameters, which involves the identity operator of the world-sheet in this unitary case, as will be explained later. Here, we expect the susceptibility to behave as $\left(\lambda-\lambda_{c}\right)^{1 / m}$. 
The simplest situation is the Ising model on a triangulated lattice $(m=3)$ : we may solve the algebraic equations (2.16) explicitly and extract the scaling behaviour of $X$. If we substitute the critical form of $X_{*}(z)=(1-z)^{m} / z$ in

$$
P(z, t)=U^{\prime}(X(z, t))-X\left(\frac{1}{z}, t\right)
$$

then claim that $U^{\prime}(\varphi)=\alpha+\beta \varphi+\gamma \varphi^{2}$, and identify different powers of $z$ ( we know that $P_{*}(z)=t_{*} z+$ higher powers of $\left.z\right)$ we may identify the critical potential:

$$
U^{\prime}(\varphi)=3-3 \varphi-\varphi^{2}
$$

and the critical value $t_{*} \equiv \lambda_{*}=10$. Now, we try to find $X$ for any $t$ :

$$
X(z, t)=\frac{\sqrt{R}}{z}+a+b z+c z^{2}
$$

again by identifying powers of $z$ in (5.2) and using $P(z, t)=\frac{t}{\sqrt{R}} z+\ldots$ This leads to the following system :

$$
\left\{\begin{aligned}
0 & =R+c \\
0 & =3 \sqrt{R}+2 a \sqrt{R}+b \\
0 & =3-4 a-a^{2}-2 b \sqrt{R} \\
\frac{t}{\sqrt{R}} & =-\sqrt{R}-3 b-2 c \sqrt{R}-2 a b
\end{aligned}\right.
$$

This way, we can express $c=-R$, eliminate $b$ so as to get a quadratic equation for $a$ with coefficients involving $R$, solve it and keep the only branch that corresponds to the critical value $a_{*}=-3$. We obtain:

$$
\begin{aligned}
& a=2(R-1)-\sqrt{4 R^{2}-2 R+7} \\
& b=-3 \sqrt{R}-2 a \sqrt{R} \\
& c=-R
\end{aligned}
$$

where $R$ is given through

$$
\lambda \equiv t=32 R^{3}-14 R^{2}+28 R-4 R(4 R-1) \sqrt{4 R^{2}-2 R+7}
$$


Finally, we obtain (implicitly) an exact expression for $X$, whose scaling behaviour is found by letting $\lambda \rightarrow \lambda_{c}$ and $z-1 \sim\left(\lambda-\lambda_{c}\right)^{\frac{1}{2 m}}$. We find the third Chebyshev polynomial (up to some arbitrary scaling on $X, z)$ which expresses $\cosh (3 \theta)$ in terms of $\cosh (\theta)$ (see the next section).

Indeed, if we consider the generic scaling limit:

$$
\begin{aligned}
& X(\lambda, z) \sim\left(\lambda-\lambda_{c}\right)^{\frac{1}{2}} \xi\left(\frac{(z-1)}{\left(\lambda-\lambda_{c}\right)^{\frac{1}{2 m}}}\right) \\
& P(\lambda, z) \sim\left(\lambda-\lambda_{c}\right)^{\frac{m+1}{2 m}} \pi\left(\frac{(z-1)}{\left(\lambda-\lambda_{c}\right)^{\frac{1}{2 m}}}\right)
\end{aligned}
$$

with $\xi$ and $\pi$ polynomials of degrees $m$ and $m+1$ respectively, and if we express the canonical Poisson bracket in terms of these scaling functions, we find 2

$$
m \xi \pi^{\prime}-(m+1) \xi^{\prime} \pi=1 .
$$

If $m=3$ we find that the Chebyshev polynomials do satisfy this relation. In any case, an obvious computation shows that hyperbolic cosines will always satisfy this scaling relation (compare next section). Will it always be the only polynomial solution?

Let us consider the $m=4$ case ( critical potentials, obtained in the same way are given in the Appendix). Again, we try to find the coefficients of successive powers of $z$ in the expression of $X(z, t)$; the equivalent to system (5.5) involves equations of higher degree, which allows for more than one solution corresponding to the expected form for $X_{*}$ : one has to choose between different branches of solutions. Among the two possible branches, only one exhibits a proper critical behaviour of the string susceptibility $R \sim\left(\lambda-\lambda_{c}\right)^{1 / 4}$. And the corresponding coordinate function behaves, in the critical vicinity, as the fourth Chebyshev polynomial. Let us note that the critical point cannot be reached by increasing the cosmological constant from zero towards its critical value because there is a pure gravity $(m=2)$ singularity on the way (for real susceptibilities).

2 This equation was also obtained in 34 
For $m=5$ the algebra becomes a bit more intricate, and among the three possible branches of solutions, two exhibit a fifth-order critical behaviour and have to be considered. One of these leads to the fifth Chebyshev polynomial for the scaling behaviour of $X$, while the other solution is not reducible to that form under rescalings of $X$ and $z$; it corresponds to

$$
\xi(T)=T^{5}+5 T^{3}+15 T .
$$

If we come back to the differential equation involving $\xi$ and $\pi$ and look for all its polynomial solutions ( degrees 5 and 6 ) we shall find Chebyshev polynomials and another solution:

$$
\begin{aligned}
& \xi(T)=T^{5}+5 T^{3}+15 T \\
& \pi(T)=T^{6}+6 T^{4}+21 T^{2}+14
\end{aligned}
$$

Its interpretation is not yet clear. It might correspond to another scaling behaviour of the matrix model around the same critical point. In any case, although $m$ is odd here, we again find maxima in the plot $\lambda$ versus $R$ which seem to hide the singularity of fifth order; again, the critical point has to be reached in a very particular way.

Having noticed the existence of this exotic solution, we may come back to the $m=4$ case, look for all possible polynomial solutions and discover that

$$
\begin{aligned}
& \xi(T)=T^{4}+4 \\
& \pi(T)=T^{5}+5 T
\end{aligned}
$$

but who has to be excluded as it corresponds to the wrong scaling $R \sim\left(\lambda-\lambda_{c}\right)^{1 / 2}$. It seems that all possible $\xi, \pi$ solutions are realized as different solutions of the algebraic equations associated with the matrix model.

Let us now turn to the non-symmetric case $U \neq V$. As we shall see in the next section, it contains all $(p, q)$ critical points (the corresponding multicritical potentials are given in the Appendix).

Now, the $(3,4)$ case reveals two branches of solution: the usual Chebyshev polynomials, and

$$
\begin{aligned}
& X \sim T^{4}-T \\
& P \sim 4 T^{3}-3
\end{aligned}
$$


which gives the proper Poisson bracket ( exotic solution, non-existing in the symmetric case) and the right critical exponent for $R$.

We also considered the $(3,5)$ case, and found two possible scaling behaviours, associated with the two possible solutions for the Poisson bracket equation in the scaling regime. Here Chebyshev polynomials are no longer possible solutions (as will be explained later) but instead:

$$
\begin{aligned}
& X=\frac{1}{3} T^{5}+5 T^{3} \pm 5 T^{2}+15 T \pm 30 \\
& Y=\frac{2}{3} T^{3}+6 T \mp 6
\end{aligned}
$$

these being two solutions of (3.2) ( to verify this, do not forget to change signs, as $P(\omega)$ scales as $Y(-\omega))$.

A solution for $P, Q, X, Y$ in the unitary case $(p=q+1)$ will be presented in the next section. The complete classification of scaling behaviours is far from being accomplished.

\section{Explicit solution of the Heisenberg relation near the $(p, q)$ critical point}

Once the scaling laws are established, it is more convenient to work with the differential form of the equations of motion (3.2). Let us use the parametrization

$$
\omega=2 \mu \cosh (\theta)
$$

Near the $(p, q)$ multicritical point, $X \sim Q \sim \mu^{q}$ and $Y \sim P \sim \mu^{p}$. We know by eq.

(3.2) that $\lambda \sim \mu^{p+q-1}$. We introduce two complete systems of polynomials in $\mu$ and $\omega$, homogeneous of degree $p$ and $q$

$$
\begin{aligned}
& \Omega_{k}^{(p)}=2 \mu^{p} \cosh (k \theta), \quad k=0,1, \ldots, p \\
& \Omega_{s}^{(q)}=2 \mu^{q} \cosh (s \theta), \quad s=0,1, \ldots, q
\end{aligned}
$$

These polynomials have Poisson brackets

$$
\left\{\Omega_{k}^{(p)}, \Omega_{s}^{(q)}\right\}=\frac{\mu^{p+q-1}}{\partial \lambda / \partial \mu}\left[(p s+q k) \frac{\sinh [(s-k) \theta]}{\sinh \theta}+(p s-q k) \frac{\sinh [(s+k) \theta]}{\sinh \theta}\right]
$$


Thus, expanding the functions $X$ and $P$ as linear combinations of (6.2)

$$
\begin{gathered}
P=\sum_{k=0}^{p} A_{k} \Omega_{k}^{(p)}, \quad X=\sum_{s=0}^{q} B_{s} \Omega_{s}^{(q)} \\
Y=\sum_{k=0}^{p}(-)^{k} A_{k} \Omega_{k}^{(p)}, \quad Q=\sum_{s=0}^{q}(-)^{s} B_{s} \Omega_{s}^{(q)}
\end{gathered}
$$

we find from (3.2) a linear system of equations for the coefficients.

This method works particularly well in the unitary case $q=m, p=m+1$. Then we have simply

$$
\begin{aligned}
& P=\Omega_{p}^{(p)}=2 \mu^{m+1} \cosh [(m+1) \theta], \\
& X=\Omega_{q}^{(q)}=\mu^{m} \cosh (m \theta), \\
& \lambda=\frac{p q}{p+q-1} \mu^{p+q-1}=(m+1) \mu^{2 m}
\end{aligned}
$$

\section{The vicinity of the critical point}

The interpretation of the momentum as the Wilson loop variable is valid also outside the critical point. In this section we will sketch the situation when the potentials $U$ and $V$ represent an arbitrary perturbation of a critical potential. For more details see the original paper by Krichever [31].

Let us parametrize the vicinity of the critical point by the coupling constants $t_{1}=\lambda, t_{2}, t_{3}, \ldots$ Then the operator $X$ will be represented in the scaling limit by a general polynomial of degree $q$. As before we introduce a "potential" $S\left(\omega, t_{1}, t_{2}, \ldots\right)$ which in our model has the meaning of the partition function of a disk without marked point [eq. (3.14)]. The function $S\left(X, t_{1}, \ldots\right)$ can be thought of as the action of a Hamiltonian system depending on the co-ordinate $X$ and the "times" $t_{m}, n=1,2, \ldots$ The total differential of the action is

$$
d S=P d X-\sum_{n} H_{n} d t_{n}
$$

where the momentum

$$
P=\frac{\partial S}{\partial X}
$$


is the Wilson loop average and $H_{n}$ are the Hamiltonians corresponding to the times $t_{m}$. By the definition (7.1) :

$$
H_{n}=-\left.\frac{\partial S}{\partial t_{n}}\right|_{X}
$$

From the commutation of the second derivatives it follows that

$$
\left.\frac{\partial P}{\partial t_{k}}\right|_{X}=-\frac{\partial H_{k}}{\partial X}, k=1,2, \ldots, q-1
$$

The relation (3.4) is a particular case of (7.4), with $k=1$. Let us mention that dispersionless KP flows are determined by the Hamiltonian equations of motion

$$
\frac{\partial P}{\partial t_{n}}=\left\{P, H_{n}\right\}, \quad \frac{\partial X}{\partial t_{n}}=\left\{X, H_{m}\right\}
$$

Each coupling constant $t_{k}$ is coupled with the corresponding scaling operator $\mathcal{O}_{k}$. Equation (7.5) implies the zero-curvature condition

$$
\frac{\partial H_{k}}{\partial t_{n}}-\frac{\partial H_{n}}{\partial t_{k}}+\left\{H_{k}, H_{n}\right\}=0
$$

In the construction of Krichever [31], the starting point was the zero-curvature condition (7.6) which is equivalent to

$$
\left.\partial_{m} H_{n}\right|_{X}=\partial_{n} H_{m} \mid X
$$

and thus implies the existence of the action such that $H_{n}=\left.\partial_{n} S\right|_{X}$.

Let us consider first the unitary case $p-m+1, q=m$. The function $H_{n}$ can be thought of as the partition function of the disk with a puncture on the world-sheet where the $\mathcal{O}_{n}$ operator is placed. For $n=1,2, \ldots, m-1$ this is the $n^{t h}$ order parameter of the model. The operator $\mathcal{P}=\mathcal{O}_{1}$ is the puncture operator corresponding to the identity operator in the conformal theory $(m, m+1)$. The dimension of the time $t_{n}$ is that of the power $\mu^{2 m+1-n}$, near the point $t_{n}=\delta_{n, 1} \mu^{2 m}$. After taking the derivative in $X$ in order to create a marked point on the boundary, according to $(\underline{7.5)}$ one obtains the loop-point correlators that were calculated in [36] for $t_{k}=\mu^{2 m} \delta_{k, 1}$ :

$$
\frac{\partial}{\partial X} H_{k}\left(X, t_{1}=\lambda, t_{2}, \ldots\right)=\mu^{k-m} \frac{\sinh (k \theta)}{\sinh (m \theta)} \sim \frac{\partial}{\partial \mu}\left(\mu^{m+k} \cosh [(m+k) \theta]\right)
$$


Integrating this formula, we find for $t_{n}=\mu^{2 m} \delta_{n, 1}$ :

$$
H_{n}=\mu^{n} \cosh (n \theta)
$$

In particular, $H_{1}=\omega$ (this is true for arbitrary $t_{k}$ ). If we interpret the quantity (7.4) in terms of an operator $\mathcal{O}_{m}$ acting on a disk with a puncture, then it should be proportional to $\lambda^{1-\gamma_{s t r} / 2-\left(1-\Delta_{m}\right)}=\lambda^{1+1 / 2 n-(1-(m-1) / 2 n)}$ and it follows that the gravitational dimension of the operator $\mathcal{O}_{k}$ coupled with the "time" $t_{k}$ is

$$
\Delta_{k}=\frac{k-1}{2 m}
$$

Therefore, the operators $\mathcal{O}_{k}, k=1,2, \ldots, m$ are the order parameters in the $(m, m+1)$ conformal field theory coupled with gravity. The operator $\mathcal{O}_{m+1}$ can be identified with the boundary operator $\partial / \partial X$ marking a point at the boundary of the loop. Note that the functions (7.8) have been interpreted in [12] as the wave-functions of the on-shell states of the closed string. They appear as leg factors in the multi-loop string amplitudes. In particular, the derivative of the two-loop correlator in the two-matrix model will be expressed as a sum of products of these functions.

In the general $(p, q)$ case, the coupling $\lambda=t_{1}$ no longer corresponds to the puncture (identity) operator, but to the operator with the minimal (negative) dimension in the model. This leads, as we have seen in the previous section, to more complicated expressions for $P$ and $X$.

There exists, however, a direction in which the solution is just as simple as that in the unitary case. It is along the coupling constant $\Lambda=t_{p-q}$ corresponding to the identity operator $\mathcal{O}_{p-q}$. It seems to us that this is the most natural definition of the non-unitary critical points. It appears when the $(p, q)$ critical points are constructed as integrable ADE models on a fluctuating lattice [36], [37] . Then the operators with negative dimensions do not appear by construction and the cosmological constant is coupled with the volume of the world-sheet. 
Along the direction $t_{k}=\delta_{k, p-q} \Lambda$ in the space of the coupling constants, the potential depends explicitly on the coupling $t=\lambda$ and the quasi-classical limit of (3.1) is no longer given by the Poisson bracket relation (3.2). Instead, the classical functions $P$ and $X$ satisfy the new Poisson bracket condition

$$
\{P, X\}_{\Lambda, \Omega} \equiv \frac{\partial P}{\partial \Lambda} \frac{\partial X}{\partial \Omega}-\frac{\partial X}{\partial \Lambda} \frac{\partial P}{\partial \Omega}=1
$$

where the new variables $\Omega$ and $\Lambda$ are related to the old ones by a non-canonical transformation

$$
\Omega=\mu^{p-q} \cosh [(p-q) \theta], \Lambda=\mu^{2 q}
$$

Equation (7.11) follows from the explicit solution for the loop average in the $(p, q)$ critical point of an ADE model on a random lattice [37]

$$
P(\omega, \Lambda)=\mu^{p} \cosh (p \theta), X(\omega, \Lambda)=\mu^{q} \cosh (q \theta) ; \quad \Lambda=\mu^{2 q}, \omega=\mu \cosh \theta
$$

\section{Boundaries and boundary operators}

In this section we will give an interpretation of the loop operators in the two-matrix model in terms of statistical systems on random surfaces.

For a string physicist $\operatorname{Tr}(x-\hat{X})^{-1}$ represents an operator creating a hole in the worldsheet of the string. In the two-matrix model (with two different potentials) there are two different loop operators

$$
W(x)=\frac{\operatorname{Tr}}{N} \frac{1}{x-\hat{X}}, \quad \tilde{W}(x)=\frac{\operatorname{Tr}}{N} \frac{1}{y-\hat{Y}}
$$

These two operators have different dimensions; their mean values read (in the unitary case $p=q+1)$

$$
\begin{aligned}
& W(x)=\left(x+\sqrt{x^{2}-\lambda}\right)^{p / q}+\left(x-\sqrt{x^{2}-\lambda}\right)^{p / q} \\
& \tilde{W}(y)=\left(y+\sqrt{\left.y^{2}-\lambda^{p / q}\right)}\right)^{q / p)}+\left(y-\sqrt{y^{2}-\lambda^{p / q}}\right)^{q / p}
\end{aligned}
$$


The experience with the ADE and SOS models on fluctuating lattices [36], [11], [37] allows usto recognize in $X$ and $Y$ the operators that create the two possible kinds of boundary in the $(p, q)$ model. It has been noticed that the ADE models exist in two phases: dense and dilute. Thus each $(p, q)=(q, p)$ model has two different statistical realizations. In one of them the boundary has dimension 1 , and in the other a non-classical dimension $p / q$. The same is true in the case of the $O(n)$ model [38]. The expansion of the two kinds of macroscopic loops in terms of local operators contains the two kinds of boundary operators in the model (see the discussion in section 2.2 of ref. [38]).

Let us illustrate this by the simplest example $p=3, q=2$. If we perform the gaussian integration in $Y$, the resulting matrix model will give the standard formulation of the $C=0$ theory $(2,3)$ (pure gravity). On the other hand, it is known [39] that this matrix model is equivalent to the $Q=1$ Potts model on a fluctuating lattice. If we choose $g_{1}=h_{1}=0, g_{2}=1, g_{3}=-g$, then the temperature $T$ and the "cosmological constant" $\beta$ are given by

$$
T=1 / \ln h_{2}, \quad \beta=\ln \left(h_{2}-1\right)-(2 / 3) \ln g
$$

Integrating over $X$ we find, in the large- $N$ limit, the following one-matrix model [39]

$$
\begin{aligned}
Z & =\int \prod_{i=1}^{N} d y_{i} e^{-\beta V_{e f f}\left(y_{i}\right)} \prod_{i \neq j}\left(y_{i}-y_{j}\right) \prod_{i, j}\left(\sqrt{a-y_{i}}+\sqrt{a+y_{j}}\right)^{-1 / 2} \\
V_{e f f}(y) & =\frac{1}{2} h_{2} \operatorname{Tr} Y^{2}+6 g \operatorname{Tr} Y+\frac{2}{3 \sqrt{g}} \operatorname{Tr} \sqrt{(a-Y)^{3}}+\frac{1}{N} \operatorname{Tr} \frac{1}{\sqrt{(a-Y)}} \operatorname{Tr} \sqrt{(a-Y)}
\end{aligned}
$$

where the constant $a$ is determined by

$$
a=\frac{1}{4 g}-\sqrt{g} \frac{\operatorname{Tr}}{N}(a-Y)^{-1 / 2}
$$

This model has the same singularity for the free energy as the standard one-matrix model for pure gravity, but different scal ing for the loop operators.

Another interesting problem is to find the amplitudes involving two kinds of boundaries

$$
W(x, y)=\left\langle\operatorname{Tr} \frac{1}{x-\hat{X}} \frac{1}{x-\hat{Y}}\right\rangle
$$

whose calculation involves the angular degrees of freedom.

Such amplitudes have been studied recently in [40]. 


\section{Two-loop correlators}

The two-point functions appear in matrix formulation as expectation values of prod-

uct of traces; $\frac{1}{N} \operatorname{Tr} X^{m}$ corresponds to a puncture operator whose action on the string world-sheet leaves a hole with a boundary of length $m$. Boundaries of any size may be considered, with the operator $\frac{1}{N} \operatorname{Tr} \frac{1}{x-\tilde{X}}$, which involves all possible punctures, weighted with the exponential of the length of their boundaries. Such an operator has well-defined expectation values, provided the one-dimensional cosmological constant $x$ is large enough. The continuum limit is then reached when we let $x$ decrease towards its critical value, so as to allow larger and larger boundaries.

There are three types of the loop-loop correlation functions in our model:

$$
\begin{gathered}
W_{X Y}(x, y)=\left\langle\operatorname{Tr} \frac{1}{x-\hat{X}} \operatorname{Tr} \frac{1}{y-\hat{Y}}\right\rangle_{c}, \\
W_{X X}\left(x_{1}, x_{2}\right)=\left\langle\operatorname{Tr} \frac{1}{x_{1}-\hat{X}} \operatorname{Tr} \frac{1}{x_{2}-\hat{X}}\right\rangle_{c} \\
W_{Y Y}\left(y_{1}, y_{2}\right)=\left\langle\operatorname{Tr} \frac{1}{y_{1}-\hat{Y}} \operatorname{Tr} \frac{1}{y_{2}-\hat{Y}}\right\rangle_{c}
\end{gathered}
$$

They may be computed in an explicit way by means of the orthogonal polynomial technique. Let us start with the first correlator (9.1). In the basis of orthogonal polynomials, we have

$$
\tilde{W}(x, y)=\frac{1}{N^{2}} \sum_{i \leq N-1, j \geq N}\left\langle i\left|\frac{1}{x-\hat{X}}\right| j\right\rangle\left\langle j\left|\frac{1}{y-\hat{Y}}\right| i\right\rangle
$$

Only the levels located near the Fermi surface $t=\lambda$ contribute to this sum, so that, in the planar limit, we can express the former matrix elements in terms of the functions $X(z), Y(z)$ : for instance, $\left\langle i+\delta\left|\frac{1}{x-\hat{X}}\right| i\right\rangle$ is the coefficient of $z^{-\delta}$ in $\frac{1}{x-X(z)}$. Thus we can express the connected two-loop function as a contour integral and perform the sum over $\delta$

$$
\begin{aligned}
W_{X Y}(x, y) & =-\frac{1}{4 \pi^{2}} \oint \oint \frac{d z d \tilde{z}}{z \tilde{z}} \sum_{\delta \geq 1} \delta(z \tilde{z})^{\delta} \frac{1}{x-X(z)} \frac{1}{y-Y(\tilde{z})} \\
& =-\frac{1}{4 \pi^{2}} \oint \oint \frac{d z d \tilde{z}}{(1-z \tilde{z})^{2}} \frac{1}{x-X(z)} \frac{1}{y-Y(\tilde{z})}
\end{aligned}
$$


where $|z|<1,|\tilde{z}|<1$ for the sum to converge.

Now, provided that $x$ and $y$ are big enough, for the equations $X(z)=x$ and $Y(z)=y$ to have unique solutions $z(x)$ and $\tilde{z}(y)$ for $|z|<1,|\tilde{z}|<1$, we calculate the contour integrals by the residua and finally obtain

$$
W_{X Y}(x, y)=\frac{z^{\prime}(x) \tilde{z}^{\prime}(y)}{(1-z(x) \tilde{z}(y))^{2}}=-\partial_{x} \partial_{y} \ln [1-z(x) \tilde{z}(y)]
$$

Note that the double pole at $z=1 / \tilde{z}$ does not contribute since it gives the total derivative under the second integral.

In a similar way we obtain the other two correlators

$$
\begin{aligned}
& W\left(x_{1}, x_{2}\right)=-\partial_{x_{1}} \partial_{x_{2}} \ln \left[\frac{z\left(x_{1}\right)-z\left(x_{2}\right)}{x_{1}-x_{2}}\right] \\
& W\left(y_{1}, y_{2}\right)=-\partial_{y_{1}} \partial_{y_{2}} \ln \left[\frac{\tilde{z}\left(y_{1}\right)-\tilde{z}\left(y_{2}\right)}{y_{1}-y_{2}}\right]
\end{aligned}
$$

We see from these formulae that the knowledge of $z(x)$ and $\tilde{z}(y)$ or, in other words, of the one-loop averages, allows the immediate reconstruction of the corresponding two-loop correlators.

Formulae (9.6) - 9.8) work, in principle, beyond the two-matrix model and are applicable to any matrix chain where the orthogonal polynomial formalism is valid.

In the scaling limit $z \equiv e^{\omega} \rightarrow 1, \omega \rightarrow 0$ we find in the unitary case $p=q+1$, using the explicit solution $(6.6)$,

$$
\begin{gathered}
\tilde{W}_{X Y}(X, Y)=-\partial_{X} \partial_{Y} \ln [\cosh (\theta)+\cosh (\tau)] \\
W_{X X}\left(X_{1}, X_{2}\right)=-\partial_{X_{1}} \partial_{X_{2}} \ln \left[\frac{\cosh \left(\theta_{1}\right)-\cosh \left(\theta_{2}\right)}{\cosh \left(q \theta_{1}\right)-\cosh \left(q \theta_{2}\right)}\right]
\end{gathered}
$$

and

$$
W_{Y Y}\left(Y_{1}, Y_{2}\right)=-\partial_{Y_{1}} \partial_{Y_{2}} \ln \left[\frac{\cosh \left(\tau_{1}\right)-\cosh \left(\tau_{2}\right)}{\cosh \left(q \tau_{1}\right)-\cosh \left(q \tau_{2}\right)}\right]
$$

where the following parametrization is used

$$
X=2 \mu^{q} \cosh (q \theta), \quad Y=2 \mu^{p} \cosh (p \tau)
$$


Expressions (9.9)- (9.11) remain true even in the non-unitary case $p-q>1$, if the deviation from the critical point is along the constant $\Lambda$ coupled with the identity operator (see the discussion at the end of section 7 ).

Let us try to relate the above results with what is known of the two-loop correlators for strings with discrete target space.

The loop-loop correlator in the one-matrix model with general potential has been calculated in [32] (see also the Appendix of [37]). In all critical regimes ( $q=2, p$ odd) it is given by

$$
W\left(X_{1}, X_{2}\right)=-\frac{\partial}{\partial X_{1}} \frac{\partial}{\partial X_{2}} \log \left(\sqrt{X_{1}+\mu^{2}}+\sqrt{X_{2}+\mu^{2}}\right)
$$

which is identical to the formula (9.10), which we obtained for the $X X$ correlator with $X=2 \cosh (\theta / 2)$. The derivative of this correlator with respect to $\lambda=\mu^{p+1}$ gives the loop-loop-point correlator and factorizes to a product of two factors

$$
\frac{\partial}{\partial \mu} W\left(X_{1}, X_{2}\right)=\frac{1}{\sqrt{X_{1}+\mu^{2}} \sqrt{X_{2}+\mu^{2}}}
$$

It has been noticed [37] that such a factorization is a general property of all strings with discrete target space. Namely, all multiloop amplitudes (three or more loops) depend on the parameters of the boundaries through a product of factors associated with loops. In our case the differentiation in $\mu$ for fixed $X_{1}, X_{2}$ gives

$$
\begin{aligned}
\mu \frac{\partial}{\partial \mu} W_{X X}\left(X_{1}, X_{2}\right) & =\frac{\partial}{\partial X_{1}} \frac{\partial}{\partial X_{2}} \frac{1}{\cosh \left(\theta_{1}\right)-\cosh \left(\theta_{2}\right)}\left[\frac{\sinh \left[(q-1) \theta_{1}\right]}{\sinh \left(q \theta_{1}\right)}-\frac{\sinh \left[(p-1) \theta_{2}\right]}{\sinh \left(p \theta_{2}\right)}\right] \\
& =\frac{\partial}{\partial X_{1}} \frac{\partial}{\partial X_{2}} \sum_{k=1}^{q-1} \frac{\sinh \left[(q-k) \theta_{1}\right]}{\sinh \left(q \theta_{1}\right)} \frac{\sinh \left[(q-k) \theta_{2}\right]}{\sinh \left(q \theta_{2}\right)}
\end{aligned}
$$

Similarly, the derivative of the $Y Y$ correlator decomposes into a sum of $p-1$ terms.

Comparing (9.15) with the standard expression for the two-loop correlator ([37], eq.(4.42)) we can express the operator $P$ in the $(p, q)$ critical regime of the two-matrix model as a linear combination of the order parameters of the corresponding string theory. 
Indeed, the loop-loop correlator diagonalizes in the momentum space $k / q, k=1,2, \ldots, q-1$, Introducing the loop operator $\Psi_{k}(X)$ with momentum $k / q$ we have

$$
\left\langle\Psi_{k}\left(X_{1}\right) \Psi_{k}\left(X_{2}\right)\right\rangle_{c}=\frac{\partial}{\partial X_{1}} \frac{\partial}{\partial X_{2}} \frac{\sinh \left[(q-k) \theta_{1}\right]}{\sinh \left(q \theta_{1}\right)} \frac{\sinh \left[(q-k) \theta_{2}\right]}{\sinh \left(q \theta_{2}\right)}
$$

This formula can be obtained from eq. (4.42) of ref. [37], or, more easily, from the Laplace image of the expression for the three-loop correlator (eq. (4.58) of ref.[37]) with one of the loops shrinked to a point. Comparing (9.15) and (9.16) we can conclude that the Heisenberg momentum $P$ can be written as a linear combination of the loop operators $\Psi_{k}(X), k=1, \ldots, q-1$. In the same way the operator $Q$ is a linear of the loop operators $\Psi_{k}(Y), k=1,2, \ldots, p-1$. Note that the $X X(Y Y)$ correlators depend only on $q(p)$. This phenomenon occurs only for the two-loop correlator and is due to the Euler characteristic of the world-sheet being zero. The two-loop average, however, contains all the operators; this is related to the fact that the Euler characteristic of the cylinder is zero. As for the mixed correlator $W_{X Y}$, its derivative with respect to $\lambda$ cannot be written in a factorized form in the non-symmetric case.

All these formulae have an especially nice interpretation in the $Z_{2}$-symmetric case of the two-matrix model $U=V$, describing the $(m, m+1)$ unitary models, which we consider in more detail in the next section.

\section{Symmetric $(V=U)$ realization of the unitary $(m, m+1)$ models}

In this case we can use the equation of motion (2.17) . The formula for the multicritical potential is given by (4.4) if one takes there $p=q=m$.

If one uses only the even potentials (polynomials in the even powers of the matrices)

one has to take, instead of eq.(4.3), the following critical expression for $X_{*}(z)$ :

$$
\frac{\partial}{\partial z} X_{*}(z)=-\frac{\left(1-z^{2}\right)^{m-1}}{z^{2}}
$$


which is the only one satisfying all the conditions of $m$-criticality and the behaviour for $z \rightarrow 0$. The expression for the multicritical potential is the same as for the general symmetric case.

Some examples of these potentials are given in the Appendix. Note that at least the even $(m=2 k)$ unitary cases provide a non-perturbatively stable definition of the corresponding rational string theory in the double scaling limit, since the corresponding multicritical potential contains the positive highest power coefficient. As far as we know, this is the simplest possible stabilization of the double scaling limit of the unitary models of gravity.

The symmetric case provides a direct statistical interpretation of the two matrix model in terms of the Ising spins on the dynamical random lattice. In this case the operator $Y$ is the $\mathbb{Z}_{2}$ image of $X$ and commutes with it. The loop-loop correlators found in the previous section correspond to the boundary conditions with fixed directions of the Ising spins: $W_{X X}$ and $W_{Y Y}$ have the same directions of the spin along the two boundaries, whereas $W_{X Y}$ has opposite directions. The $\mu$-derivative of the loop-loop correlators factorizes both for $W_{X X}=W_{Y Y}$ and $W_{X Y}$ :

$$
\begin{gathered}
\mu \frac{\partial}{\partial \mu} W_{X X}\left(X_{1}, X_{2}\right)=\frac{\partial}{\partial X_{1}} \frac{\partial}{\partial X_{2}} \sum_{k=1}^{m-1} \frac{\sinh \left(k \theta_{1}\right)}{\sinh \left(m \theta_{1}\right)} \frac{\sinh \left(k \theta_{2}\right)}{\sinh \left(m \theta_{2}\right)} \\
\mu \frac{\partial}{\partial \mu} W_{X Y}\left(X_{1}, X_{2}\right)=\frac{\partial}{\partial X_{1}} \frac{\partial}{\partial X_{2}} \sum_{k=1}^{m-1}(-)^{k} \frac{\sinh \left(k \theta_{1}\right)}{\sinh \left(m \theta_{1}\right)} \frac{\sinh \left(k \theta_{2}\right)}{\sinh \left(m \theta_{2}\right)}
\end{gathered}
$$

where

$$
X_{1}=\mu^{m} \cosh \left(m \theta_{1}\right), X_{2}=\mu^{m} \cosh \left(m \theta_{2}\right)
$$

We see that the two-point correlator diagonalizes if we pass to the operators imposing symmetric $(+)$ and antisymmetric $(-)$ boundary conditions. 


\section{Discussion}

We made an attempt to explicitly work out the idea proposed by M. Douglas about the possibility to represent all models of $(p, q)$ rational matter interacting with $2 \mathrm{D}$ gravity as the multicritical regimes of the two matrix model, which describes random planar graphs of specially adjusted statistics with the Ising spins on it. Although it should be clear to the reader that this purpose has not been achieved completely in our paper, we made a few steps forward in this direction, leaving many other important details for the future.

We determined the pairs of $(p, q)$ multicritical potentials, i.e. the sets of coupling constants ("temperatures") rendering the $(p, q)$ matter critical. By shifting the cosmological coupling away from its critical value we reconstructed the known scaling solutions for the one loop averages in these models.

We found an intriguing relation between the canonical momentum and the loop average in the dispersionless (large $N$ ) limit of the generalized KdV formalism.

The two-loop correlators are given in a closed form, which is valid for arbitrary potentials. Some of our formulae and the method of calculation apply for any chain of matrices admitting orthogonal polynomial representation. The scaling form of loop-loop correlators is compatible with the previous results obtained for the RSOS models coupled with gravity: in the unitary $(m, m+1)$ model the correlator can be factorized into $(m-1)$ terms describing the corresponding finite number of excitations (corresponding to the order operators) propagating between the macroscopic states (loops).

One of the most attractive features of the construction of the theory of $(p, q)$ matter coupled with $2 \mathrm{D}$ gravity via the two matrix model, is the explicit $(p, q)$ versus $(q, p)$ duality. This last property is far from evident in the standard construction based on the RSOS models on a random lattice.

Among the problems to be addressed further are the following:

1.The calculation of the multi-loop correlators in form as explicit as was done for the one matrix model. 
2. The description of the double scaling limit and of the topological $1 / N$ expansion, using the exact (for any $N$ ) operator equations (2.15). This becomes especially interesting in view of the possibility to give within this model a nonperturbative stable definition of the double scaling limit of $(2 n, 2 n+1)$ unitary models (note that the critical potentials in the Appendix (even case) have a positive coefficient in front of the highest power for $m=2 n)$.

3. It would be interesting to compare the $1 / N$ expansion in the two matrix model with the "surface surgery" procedure worked out for the ADE models on random surfaces [37. In particular, it would be very instructive to reproduce the open string amplitudes found recently in the framework of the SOS model [40]. In the two matrix model they must correspond to the loop amplitudes with the mixed boundary (including the products of both matrices) in the loop operator. Some recent results in this direction were obtained in [41] using the methods of ref. [42].

4. A possible application of the formalism worked out here could be the investigation of the critical regimes of the so-called induced gauge theory [29] in the large N limit.

\section{Acknowledgements}

We thank J.Alfaro, D.Boulatov, E.Brezin, M.Douglas, I. Krichever and A.Migdal for discussions. 


\section{Appendix A. (p,q) Multicritical Potentials for the Two Matrix Model}

\section{A.1. Nonsymmetric case}

$$
\begin{aligned}
& q=2, p=3: \quad V(y)=y-\frac{y^{2}}{2} \\
& U(x)=-x+\frac{x^{2}}{2}+\frac{x^{3}}{3} \\
& q=3, p=4: \quad V(y)=-5 y+\frac{5 y^{2}}{2}+\frac{y^{3}}{3} \\
& U(x)=5 x+\frac{5 x^{3}}{3}-\frac{x^{4}}{4} \\
& q=4, p=5 \quad V(y)=11 y-\frac{11 y^{2}}{2}+\frac{11 y^{3}}{3}-\frac{y^{4}}{4} \\
& U(x)=-11 x+\frac{22 x^{3}}{3}+\frac{11 x^{4}}{4}+\frac{x^{5}}{5} \\
& q=2, p=4 \quad V(y)=2 y+\frac{y^{2}}{2} \\
& U(x)=2 x-\frac{x^{2}}{2}+\frac{2 x^{3}}{3}+\frac{x^{4}}{4} \\
& q=3, p=5: \quad V(y)=-7 y-\frac{7 y^{2}}{2}+\frac{y^{3}}{3} \\
& U(x)=-7 x+\frac{7 x^{3}}{3}-\frac{7 x^{4}}{4}+\frac{x^{5}}{5} \\
& q=5, p=7: \quad V(y)=-23 y-23 y^{2}+\frac{115 y^{3}}{3}-\frac{23 y^{4}}{4}+\frac{y^{5}}{5} \\
& U(x)=-23 x+\frac{115 x^{3}}{3}-\frac{345 x^{4}}{4}+\frac{161 x^{5}}{5}-\frac{23 x^{6}}{6}+\frac{x^{7}}{7}
\end{aligned}
$$

A.2. Symmetric case $U=V=V_{m}$ describing the $(m, m+1)$-unitary models $p=q=m$

$$
\begin{aligned}
& V_{3}(x)=-3 x-\frac{3 x^{2}}{2}+\frac{x^{3}}{3} \\
& V_{4}(x)=8 x+2 x^{2}+\frac{8 x^{3}}{3}+\frac{x^{4}}{4} \\
& V_{5}(x)=-15 x-\frac{5 x^{2}}{2}+15 x^{3}-\frac{15 x^{4}}{4}+\frac{x^{5}}{5} \\
& V_{6}(x)=24 x+3 x^{2}+\frac{224 x^{3}}{3}+39 x^{4}+\frac{24 x^{5}}{5}+\frac{x^{6}}{6}
\end{aligned}
$$


A.3. Even Symmetric Potentials $V_{m}$ for $(m, m+1)$ models

$$
\begin{aligned}
& V_{3}(x)=2 x^{2}-\frac{x^{4}}{12} \\
& V_{4}(x)=11 x^{2}-x^{4}+\frac{x^{6}}{30} \\
& V_{5}(x)=\frac{452 x^{2}}{5}-13 x^{4}+\frac{4 x^{6}}{5}-\frac{x^{8}}{56} \\
& V_{6}(x)=\frac{64475 x^{2}}{63}-\frac{604 x^{4}}{3}+\frac{158 x^{6}}{9}-\frac{5 x^{8}}{7}+\frac{x^{10}}{90}
\end{aligned}
$$




\section{References}

[1] A.M.Polyakov, Phys. Lett.B 103 (1981) 207

[2] F.David, Nucl. Phys. B 257 [FS14] (1985)45

[3] V.A.Kazakov, Phys. Lett.B 150 (1985) 282

[4] V.A.Kazakov, I.K.Kostov and A.A.Migdal, Phys. Lett.B 157 (1985) 295

[5] V.A.Kazakov, Phys. Lett.A 119 (1986) 140; D.V.Boulatov and V. Kazakov, Phys. Lett.B 186 (1987) 379

[6] V.A.Kazakov and A.A.Migdal, Nucl. Phys. B 311 (1989) 181

[7] I.K.Kostov, Mod. Phys. Lett. A4 (1989) 217

[8] V.Knizhnik, A.Polyakov and A.Zamolodchikov, Mod. Phys. Lett. A3 (1988) 819

[9] F.David, Mod. Phys. Lett. A3 (1988) 1651

[10] J.Distler and H.Kawai, Nucl. Phys. B 321 (1989) 509

[11] I.K.Kostov, Proceedings of the Cargèse Workshop 1990; Phys. Lett.266 B (1991) 42 and 317; Nucl. Phys. B 376 (1992) 539

[12] G.Moore, N.Seiberg and M.Staudacher, Nucl. Phys. B 362 (1991) 665

[13] M. Goulian, Phys. Lett.264B (1991) 292

[14] P.Di Francesco and D.Kutasov, Nucl.Phys. 342 (1990) 589

[15] M. Goulian and M. Lee, Phys. Rev. Lett.66 (1991) 2051

[16] V.Dotsenko, Proceedings of the Cargèse meeting 1990

[17] M.Douglas and S.Shenker, Nucl. Phys. B 335 (1990), E.Brezin and V.Kazakov, Phys. Lett.236B (1990) 144, D.Gross and A.A.Migdal, Phys. Rev. Lett.64 (1990) 127

[18] M.Douglas, Phys. Lett.238B (1990) 176

[19] I.K.Kostov, Gauge-invariant matrix model for the A-D-E closed strings, Phys. Lett.297B (1992) 74

[20] V.Kazakov, Mod. Phys. Lett. A4 (1989) 2125

[21] M. Staudacher, Nucl. Phys. B 336 (1990) 349

[22] E.Brezin, M.Douglas, V.A.Kazakov and S.Shenker, Phys. Lett.237B (1990) 43

[23] C.Itzykson and J.-B.Zuber, J. Math. Phys. 21 (1980) 411

[24] M.L.Mehta, Comm. Math. Phys. 79 (1981) 327

[25] M.Douglas, Proceedings of the Cargèse Workshop, 1990

[26] T. Tada, Phys.Lett. B259 (1991) 442, S. Odake, Phys. Lett.269 (1991) 300, T. Tada and T. Yamaguchi, Phys. Lett.250 (1990) 38,

[27] Chadha, Machoux, M.-L. Mehta, J. Phys. A: Math. Gen. 14 (1981) 579

[28] E.Martinec, Comm. Math. Phys.138 (1991) 437; S. Ryang, Phys. Rev. D46 (1992) 1873; A. Marshakov, A. Mironov and A. Morozov, Mod. Phys. Lett.A7 (1992) 1345; E. Gava and K. Narain, Phys. Lett.B263 (1991) 213

[29] V.A.Kazakov, preprint CERN-TH.6754/92, November 1992, to appear in Nucl.Phys.B, Proceedings of the Conference "Lattice-92", Amsterdam (1992) 
[30] Jan de Boer,Nucl. Phys. B336 (1991) 602

[31] I.Krichever, preprint LPTENS-92/18 (May 1992)

[32] J.Ambjorn, A.Krzyvicki and Yu.M.Makeenko, Phys. Lett.B251 (1990) 517

[33] D. Gross and A.A. Migdal, Nucl. Phys. B 340 (1990) 333

[34] B. Eynard and J. Zinn-Justin, Preprint SPhT/92-163

[35] I.K. Kostov, Phys. Lett.266 (1991) 42

[36] I. Kostov, Nucl. Phys. B 326 (1989)583

[37] I. Kostov, Nucl. Phys. B 376 (1992) 539

[38] I. Kostov and M. Staudacher, Nucl. Phys. B 384 (1992) 459

[39] V. Kazakov and I. Kostov, unpublished; I. Kostov, in Jaca 1988, Proceedings, Nonperturbative aspects of the standard model, 295

[40] V. Kazakov and I. Kostov, Nucl. Phys. B 386 (1992) 520

[41] M. Staudacher, Combinatorial solution of the two matrix model, Preprint RU-92-64, January 1993

[42] J.Alfaro, preprint CERN-TH-6531/92, July 1992 